\title{
Small amounts of pesticides reaching target insects
}

\author{
David Pimentel • Michael Burgess
}

Received: 27 August 2011/Accepted: 12 September 2011/Published online: 24 September 2011

(C) Springer Science+Business Media B.V. 2011

About 545 million $\mathrm{kg}$ of pesticides are applied to US crops each year: $20 \%$ insecticides; $68 \%$ herbicides; and $12 \%$ fungicides for pest control. Despite this heavy and costly application of pesticides, pests destroy an estimated $37 \%$ of all US crops while the treated crop losses worldwide are about 50\%. In the US, the Environmental Protection Agency reports an estimated 300,000 human pesticide poisonings as a part of the cost of applying pesticides. Worldwide, the number of serious pesticide poisonings is much higher, as Richter (2002) reports 26 million human pesticide poisonings with 220,000 deaths occur each year.

Approximately 50\% of US pesticides are applied by aircraft. The problem with aerial application of pesticides is that only about $50 \%$ of the pesticides applied ever reach the target crops. To combat the pest insects and plant pathogens, a protective coating of the pesticides has to be placed on the susceptible portions of the crop plants. The amount of pesticides reaching pests, like insects, is generally an extremely small percentage of the pesticide applied. For example, in a study of the insecticide consumed by the cabbage white butterfly (Pieris rapae) caterpillars in our collard plots documented that only about $0.003 \%$ of the $1 \mathrm{~kg} / \mathrm{ha}$ of insecticide applied was consumed by the target $P$. rapae caterpillars (Pimentel 1995). This calculation was based on an infestation of 150,000 caterpillars per hectare with each caterpillar eating $0.1 \mathrm{~cm}^{2} /$ day and included factors for plant leaf area and pesticide drift (Pimentel 1995).

This $0.003 \%$ of the insecticide reaching the cabbage pests parallels the amount of pesticide reaching pests in other crops. An investigator reported that the aphids in the field of beans collected only $0.03 \%$ of the insecticides applied. In another study with mirid insects on cocoa, the percentage was nearly the same or $0.02 \%$ of the applied insecticide reaching the mirids (Pimentel 1995). An additional example of the small amount of insecticide actually reaching target insects on cotton was with the Heliothis caterpillar.

Readers should send their comments on this paper to BhaskarNath@aol.com within 3 months of publication of this issue.

D. Pimentel $(\bowtie) \cdot$ M. Burgess

Cornell University, Ithaca, NY, USA

e-mail:dp18@cornell.edu 
In this study, the investigator reported that only $0.0000001 \%$ of the insecticide reached these pests (Pimentel 1995). Even under ideal laboratory conditions, where 200 insecticide spray droplets averaging 53 microns were applied to each square centimeter of leaf surface, only $10 \%$ of red mite spider eggs on the leaves were hit by the spray droplets (Pimentel 1995). Thus, it is nearly impossible to control insect and mite pests on crops by applying the spray insecticide directly to the target pests.

To spray and hit aerial flying insects, like mosquitoes, is even more difficult because the spray droplets must be only from 2- to 16-microns in diameter. Such small insecticide droplets are not only difficult to produce but many will drift beyond the target area, never touching the target mosquitoes. Based on the estimate that target mosquitoes only receive about $0.0000001 \%$ of the aerial spray (Pimentel 1995), 1 million insecticide droplets must be produced to hit one target mosquito. Suppose a city spent $\$ 500,000$ for mosquito control and only 1 insecticide droplet in a million hits a mosquito. This means that the $50 \notin$ of the tax money resulted in killing 1 mosquito.

\section{References}

Pimentel, D. (1995). Amounts of pesticides reaching target pests: Environmental impacts and ethics. Journal of Agricultural and Environmental Ethics, 8, 17-29.

Richter, E. D. (2002). Acute pesticide poisonings. In D. Pimentel (Ed.), Encyclopedia of pest management (1st ed., pp. 3-6). Boca Raton: CRC Press, A Taylor \& Francis Group. 